\title{
Experimental Designs for Model Discrimination
}

\author{
FRIEDRICH PUKELSHEIM and JAMES L. ROSENBERGER
}

\begin{abstract}
We present designs which perform well for several objectives simultaneously. Three different approaches are discussed: to augment a given design in an optimal way, to evaluate a mixture of the various criteria, and to optimize one objective subject to achieving a prescribed efficieny level for the others. Our sample designs are for the situation of discriminating between a second- and a third-degree polynomial fit, under the D-criterion and geometric mixtures of D-criteria.
\end{abstract}

KEY WORDS: Augmentation designs; mixture designs; designs with guaranteed efficiencies; D-optimality; general equivalence theorem.

\section{INTRODUCTION}

Many results on optimal experimental designs are derived under the assumption that, at the design stage, the statistical model is known. More often than not this is not the case. Indeed, the experimenter's goal often is to implement a design that is efficient for two or more models that might fit the experiment, in order to discriminate between them and then selects the one which describes the situation best. For an early exposition of the issue see the seminal paper Stigler (1971), or the discussion paper Atkinson and Cox (1974). Practical settings where the problem arises may be found, for example, in Hunter and Reiner (1965), Cook and Nachtsheim (1982).

In this paper we review several solutions to the problem that have been proposed in the literature. The examples we list have in common that all of them may be derived from a single equivalence theorem in the spirit of Kiefer and Wolfowitz (1960), thus pulling together seemingly divergent appraoches. All of them rely on maximizing some sort of information, that is, minimizing a function of the variance-covariance matrix of the leastsquares estimator. Thus these solutions are complementary to the approach taken by Box and Draper (1959) whose designs optimize a mixture of variance and squared bias.

Friedrich Pukelsheim is Professor, Institut für Mathematik, Universität Augsburg, D-8900 Augsburg, Germany. James L. Rosenberger is Associate Professor and Head, Department of Statistics, The Pennsylvania State University, University Park, PA 16802. This article was written while the first author was visiting PSU. The authors thank Paul D. Johnson for helping with the computations. 
As a specific example with multiple objectives, we consider the discrimination between a second-degree and a third-degree polynomial fit model. Suppose the experimental runs are determined by a single variable

$$
x \in[-1,1] .
$$

The experimenter hopes that a second-degree polynomial model adequately describes the expected observations $Y_{x}$,

$$
\mathrm{E}\left(Y_{x}\right)=\theta_{0}+\theta_{1} x+\theta_{2} x^{2} .
$$

Yet it is desirable to guard against a third-degree model,

$$
\mathrm{E}\left(Y_{x}\right)=\theta_{0}+\theta_{1} t+\theta_{2} x^{2}+\theta_{3} x^{3} .
$$

This calls for a test, in a third-degree model, of whether the parameter $\theta_{3}$ of the cubic term vanishes or not. If there is significant evidence that $\theta_{3}$ is not zero then the thirddegree model (3) is entertained, with parameter vector $\theta_{(3)}=\left(\theta_{0}, \theta_{1}, \theta_{2}, \theta_{3}\right)^{\prime}$. Otherwise the second-degree model (2) is employed, with parameter vector $\theta_{(2)}=\left(\theta_{0}, \theta_{1}, \theta_{2}\right)^{\prime}$.

This setting gives rise to the following design problem. Find experimental designs that efficiently serve all three objectives simultaneously,

i) to discriminate between models (2) and (3) and, depending on the decision,

ii) to make inferences in model (2),

iii) or in model (3).

We review three approaches to the problem that have been proposed in the literature. The resulting designs are compared through the efficiencies that they have for each of the objectives i-iii, as listed in Table 1.

The efficiencies are defined as follows. Denoting by $\xi^{*}$ the D-optimal design for the $k$-dimensional parameter vector $\theta$, any other design $\xi$ has D-efficiency

$$
\operatorname{D-eff}(\xi)=\frac{[\operatorname{det} M(\xi)]^{1 / k}}{\left[\operatorname{det} M\left(\xi^{*}\right)\right]^{1 / k}} .
$$

Here $M(\xi)$ denotes the $k \times k$ moment matrix of the design $\xi$. The scaling with the $k$ th root makes the criterion homogeneous of degree one. We assume throughout that the usual determinant criterion is appropriate to evaluate the individual objectives.

In Section 2 we first list the D-optimal designs 2.1, 2.2, 2.3 separately for each of the three goals i, ii, iii. 
Table 1. Efficient designs for second- and third-degree model discrimination

Section Design Efficiencies for $\theta_{3} \quad \theta_{(3)} \quad \theta_{(2)}$

$\begin{array}{lllllll}2.1 & \left.\text { optimal for } \theta_{3} \text {, on }[-1,1] \quad \text { (optimal value } 0.06250\right) & 1 & .93 & .75\end{array}$

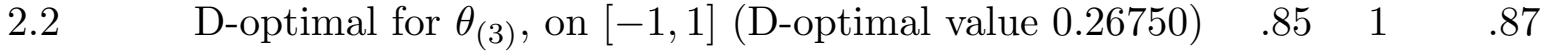

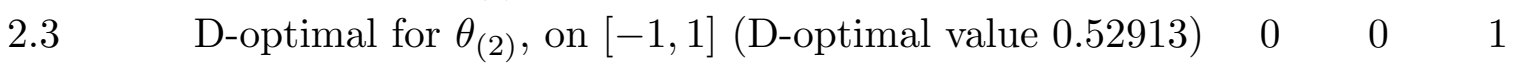

$\begin{array}{lllll}3.1 & \text { uniform, on the five points } \pm 1, \pm 1 / 2,0 & .72 & .94 & .84\end{array}$

$\begin{array}{lllll}3.2 & \text { half D-optimally augmented for } \theta_{(2)} \text {, on }[-1,1] & .42 & .89 & .94\end{array}$

4.1 mixture D-optimal for $\theta_{(2)}$ and $\theta_{(3)}$, on $[-1,1] \quad$. $\quad \begin{array}{llll}66 & .98 & .91\end{array}$

$\begin{array}{llllll}4.2 & \text { mixture D-optimal for } \theta_{(2)} \text { and } \theta_{(3)}, \text { on } \pm 1, \pm 1 / 2,0 & .64 & .96 & .90\end{array}$

$\begin{array}{lllll}4.3 & \text { mixture D-optimal for } \theta_{(2)} \text { and } \theta_{3} \text {, on } \pm 1, \pm 1 / 2,0 & 1.00 & .94 & .75\end{array}$

$\begin{array}{llllll}5.1 & \text { D-optimal for } \theta_{(2)}, 50 \% \text { efficient for } \theta_{3} \text {, on }[-1,1] & .5 & .93 & .94\end{array}$

$\begin{array}{lllll}5.2 & \text { D-optimal for } \theta_{(2)}, 50 \% \text { efficient for } \theta_{3} \text {, on } \pm 1, \pm 1 / 2,0 & .5 & .92 & .93\end{array}$

NOTE: Efficiencies for the individual component $\theta_{3}$ are evaluated in the third-degree model, as are the D-efficiencies for the full parameter vector $\theta_{(3)}=\left(\theta_{0}, \theta_{1}, \theta_{2}, \theta_{3}\right)^{\prime}$. The D-efficiencies for $\theta_{(2)}=\left(\theta_{0}, \theta_{1}, \theta_{2}\right)^{\prime}$ are calculated in the second-degree model, with the exception of 4.3 where both efficiencies are computed in a third-degree model. 
In Section 3 we discuss optimal augmentation of a given design (Covey-Crump and Silvey 1970; Wynn 1977, 1982; Welch 1982; Chaloner 1984). The uniform equispaced design 3.1 which assigns the same number of observations to the five equispaced points $-1,-1 / 2,0,1 / 2,1$ has some intuitive appeal, apart from any optimality criterion. The augmentation design 3.2 takes half of the observations from the given design 3.1 and adjoins the other half in a way which is D-optimal for $\theta_{(2)}$.

Section 4 presents designs which maximize the mixture of two criteria (Läuter 1974, 1976; Lau and Studden 1985; Bunke and Bunke 1986; Lim and Studden 1988; Dette 1991). Design 4.1 mixes the D-criterion for $\theta_{(2)}$ and the D-criterion for $\theta_{(3)}$, on the experimental domain $[-1,1]$ (Dette 1990). Design 4.2 evaluates the same objective function, but is restricted to the five equispaced points $\pm 1, \pm 1 / 2,0$. Design 4.3 mixes the criterion for the individual parameter $\theta_{3}$ and the D-criterion for $\theta_{(2)}$, again restricted to the five points $\pm 1, \pm 1 / 2,0$.

In Section 5 we impose the constraint that our solution must be at least 50 percent efficient for the individual component $\theta_{3}$ (Stigler 1971; Studden 1982; Lee 1987, 1988; Lau 1988). We present two designs both of which are constrained D-optimal for $\theta_{(2)}$. For design 5.1 (Studden 1982) the experimental domain is $[-1,1]$. Design 5.2 is again restricted to the equispaced points $\pm 1, \pm 1 / 2,0$.

Of course, the designs presented here are by no means exhaustive. There are many other designs that satisfy the same purpose, and there are other approaches with more emphasis on nonlinear modelling and sequential designs (Atkinson and Fedorov 1975a,b). Our discussion in Section 6 provides some guidance to make a choice. However, the final selection will reflect the peculiarities of the experimental situation under study, or the peculiarities of the experimenter, or those of the statistician.

The derivation of these designs, seemingly as diverse as the literature is scattered, may be unified using an appropriate generalization of the celebrated equivalence theorem of Kiefer and Wolfowitz (1960). The pertinent arguments are sketched in the Appendix, following Pukelsheim (1980, 1992).

\section{INDIVIDUAL OPTIMALITY}

Our designs $\xi$ are given in the form $\xi\left(x_{i}\right)=w_{i}$, on no more than five support points $x_{i} \in[-1,1]$, and with positive weights $w_{i}$ summing to one. Such a design $\xi$ directs the experimenter to draw a fraction $w_{i}$ of all observations under experimental conditions $x_{i}$. 
Assuming that the observations from model (3) are uncorrelated and homoscedastic, the performance of a design $\xi$ depends on its $4 \times 4$ third-degree moment matrix $M_{3}(\xi)$, with entries $m_{p, q}=\mu_{p+q-2}=\sum_{i} w_{i} x_{i}^{p+q-2}$ for $p, q=1,2,3,4$. In model (2), we evaluate of $M_{3}(\xi)$ the $3 \times 3$ top left subblock, the second-degree moment matrix $M_{2}(\xi)$. Each of our designs is symmetric around zero, and so all the odd moments vanish.

As is usual in the approximate design theory (Kiefer 1974), every probability distribution $\xi$ on $[-1,1]$ with a finite support is called a design and competes for optimality. A design is optimal for $\theta_{3}$ when it minimizes the bottom right entry of $M_{3}(\xi)^{-1}$ among all designs $\xi$. A design is D-optimal for $\theta_{(3)}$ when it maximizes the determinant of $M_{3}(\xi)$. It is D-optimal for $\theta_{(2)}$ when maximizing the determinant of $M_{2}(\xi)$. In addition to the designs we display the moment matrix and its inverse, $M_{3}(\xi)$ and $M_{3}(\xi)^{-1}$, as a means to see how the lower order moments of $\xi$ determine its performance.

Since more than one optimality criterion is of interest we must standardized them so as to enable a meaningful comparison. This is achieved by turning to

i) $\left[\left(M_{3}(\xi)^{-1}\right)_{44}\right]^{-1}$ for $\theta_{3}$,

ii) $\left[\operatorname{det} M_{3}(\xi)\right]^{1 / 4}$ for $\theta_{(3)}$, and

iii) $\left[\operatorname{det} M_{2}(\xi)\right]^{1 / 3}$ for $\theta_{(2)}$.

As a function of the moment matrices, the criteria then are all positively homogeneous, concave, and take value one for the identity matrix. Since they are information functions as defined in Pukelsheim (1980), we call the associated optimal values the optimal information.

\subsection{Optimal Design for $\theta_{3}$}

In a third-degree model, the optimal design $\xi$ for the individual component $\theta_{3}$ maximizes the criterion $\left[\left(M_{3}(\xi)^{-1}\right)_{44}\right]^{-1}$, and is supported by the Chebyshev points $\pm 1, \pm 1 / 2$ (Kiefer and Wolfowitz 1959). The weights, the third-degree moment matrix, and its inverse are

$$
\begin{gathered}
\xi( \pm 1)=1 / 6, \quad \xi( \pm 1 / 2)=1 / 3 \\
M_{3}(\xi)=\left(\begin{array}{cccc}
1 & \cdot & 0.5 & \cdot \\
\cdot & 0.5 & \cdot & 0.38 \\
0.5 & \cdot & 0.38 & \cdot \\
\cdot & 0.38 & \cdot & 0.34
\end{array}\right), \quad M_{3}(\xi)^{-1}=\left(\begin{array}{cccc}
3 & \cdot & -4 & \cdot \\
\cdot & 11 & \cdot & -12 \\
-4 & \cdot & 8 & \cdot \\
\cdot & -12 & \cdot & 16
\end{array}\right) .
\end{gathered}
$$

Dots indicate zeros. The optimal information for $\theta_{3}$ is $1 / 16=0.0625$. 


\subsection{D-optimal Design for $\theta_{(3)}$}

In a third-degree model, the D-optimal design for the full vector $\theta_{(3)}$, maximizing $\left[\operatorname{det} M_{3}(\xi)\right]^{1 / 4}$, is (Kiefer 1959)

$M_{3}(\xi)=\left(\begin{array}{cccc}1 & \cdot & 0.6 & \cdot \\ \cdot & 0.6 & \cdot & 0.52 \\ 0.6 & \cdot & 0.52 & \cdot \\ \cdot & 0.52 & \cdot & 0.50\end{array}\right), \quad M_{3}(\xi)^{-1}=\left(\begin{array}{cccc}3.25 & . & -3.75 & \cdot \\ \cdot & 15.75 & . & -16.25 \\ -3.75 & \cdot & 6.25 & \cdot \\ \cdot & -16.25 & . & 18.75\end{array}\right)$.

The D-optimal information for $\theta_{(3)}$ is $2 / 5^{5 / 4}=0.26750$.

\subsection{D-optimal Design for $\theta_{(2)}$}

In a second-degree model, the D-optimal design $\xi$ for the vector $\theta_{(2)}$, maximizing $\left[\operatorname{det} M_{2}(\xi)\right]^{1 / 3}$, is $\xi( \pm 1)=1 / 3, \xi(0)=1 / 3$. The D-optimal information for $\theta_{(2)}$ is $4^{1 / 3} / 3=$ 0.52913 . Under this design, in a third-degree model, neither the vector $\theta_{(3)}$ nor the component $\theta_{3}$ are estimable.

\section{OPTIMAL AUGMENTATION DESIGNS}

\subsection{Uniform Equispaced Design}

A design with some appeal of symmetry and balancedness is the uniform design $\xi_{0}$ on five equispaced points,

$\xi_{3}( \pm 1)=\xi_{0}( \pm 1 / 2)=\xi_{0}(0)=1 / 5$,
$\left.M_{0}\right)=\left(\begin{array}{cccc}1 & \cdot & 0.5 & \cdot \\ \cdot & 0.5 & \cdot & 0.43 \\ 0.5 & \cdot & 0.43 & \cdot \\ \cdot & 0.43 & \cdot & 0.41\end{array}\right), M_{3}\left(\xi_{0}\right)^{-1}=\left(\begin{array}{cccc}2.43 & \cdot & -2.86 & \cdot \\ \cdot & 18.06 & \cdot & -18.89 \\ -2.86 & \cdot & 5.71 & \cdot \\ \cdot & -18.89 & \cdot & 22.22\end{array}\right)$.

It has respective efficiencies of 72,94 , and 84 percent for $\theta_{3}, \theta_{(3)}$, and $\theta_{(2)}$.

Of course, there is no direct merit in the constant spacing. The high efficiencies are explained by the fact that the points $\pm 1, \pm 1 / 2,0$ are the second- and third-degree Chebyshev points (Kiefer and Wolfowitz 1959; Studden 1968). They already appear as support points for the optimal designs 2.1 and 2.3, and are close to the support points of design 2.2 . 


\subsection{D-Optimal Augmentation for $\theta_{(2)}$}

For the second-degree model, the previous design $\xi_{0}$ has a D-efficiency of 84 percent for $\theta_{(2)}$. As an alternative, only half of the observations are drawn according to the old design $\xi_{0}$. Subject to this 'protected' design portion, the other half is filled in a D-optimal way for $\theta_{(2)}$. That is, the criterion to maximize is

$$
\left\{\operatorname{det}\left[\frac{1}{2} M_{2}\left(\xi_{0}\right)+\frac{1}{2} M_{2}(\xi)\right]\right\}^{1 / 3}
$$

The resulting design is

$$
\begin{gathered}
\xi( \pm 1)=.2987, \quad \xi( \pm 1 / 2)=.1, \quad \xi(0)=.2026 \\
M_{3}(\xi)=\left(\begin{array}{cccc}
1 & \cdot & 0.65 & \cdot \\
\cdot & 0.65 & \cdot & 0.61 \\
0.65 & \cdot & 0.61 & \cdot \\
\cdot & 0.61 & \cdot & 0.60
\end{array}\right), M_{3}(\xi)^{-1}=\left(\begin{array}{cccc}
3.22 & \cdot & -3.42 & \cdot \\
\cdot & 35.74 & \cdot & -36.30 \\
-3.42 & \cdot & 5.26 & \cdot \\
\cdot & -36.30 & . & 38.52
\end{array}\right) .
\end{gathered}
$$

The design has respective efficiencies of 42,89 , and 94 percent for $\theta_{3}, \theta_{(3)}$, and $\theta_{(2)}$. Thus the D-efficiency for $\theta_{(2)}$ has increased by 10 percent, at the cost of the other two efficiencies, particular that for $\theta_{3}$.

We sketch a proof of optimality along the outline in the Apendix. To see that $\xi$ is the D-optimal augmentation for $\theta_{(2)}$ of the old design $\xi_{0}$, we represent it as $\xi=$ $\left(\xi_{0}+\xi_{w}\right) / 2$ with $\xi_{w}( \pm 1)=w, \xi_{w}(0)=1-2 w$. That is, the new part $\xi_{w}$ is supported by the three second-degree Chebyshev points $\pm 1,0$. The equivalence theorem for this situation (Wynn 1977, Welch 1982) rests on the evaluation of the polynomial $P(x)=\left(1, x, x^{2}\right)^{\prime}\left(M_{2}\left(\xi_{0} / 2+\xi_{w} / 2\right)\right)^{-1}\left(1, x, x^{2}\right)$, which gives the standardized variances of the estimated response surface. Optimality of $\xi$ requires $P( \pm 1)=P(0)$, necessitating $w=(1+\sqrt{71 / 5}) / 12=.3974$. With this value, we find

$$
P(x)=3.2-5.3 x^{2}+5.3 x^{4}
$$

Now $P(x) \leq 3.2=P(0)=P( \pm 1)$, for all $x \in[-1,1]$, establishes the optimality of $\xi$.

\section{OPTIMAL MIXTURE DESIGNS}

The objective in this section is to optimize the geometric mean of the design criteria. Of course, the criteria may also be averaged by the arithmetic mean, or the harmonic mean (Cook and Nachtsheim 1982). 
4.1 D-Optimal Mixture Designs for $\theta_{(2)}$ and $\theta_{(3)}$, on $[-1,1]$

The geometric mean of the D-criteria for $\theta_{(2)}$ and $\theta_{(3)}$ is

$$
\left\{\left[\operatorname{det} M_{2}(\xi)\right]^{1 / 3}\left[\operatorname{det} M_{3}(\xi)\right]^{1 / 4}\right\}^{1 / 2}
$$

The optimal design with respect to this objective function is (Dette 1990)

$$
M_{3}(\xi)=\left(\begin{array}{cccc}
1 & \cdot & 0.63 & \cdot \\
\cdot & 0.63 & \cdot & 0.58 \\
0.63 & \cdot & 0.58 & \cdot \\
\cdot & 0.58 & \cdot & 0.57
\end{array}\right), M_{3}(\xi)^{-1}=\left(\begin{array}{cccc}
3.21 & \cdot & -3.51 & \cdot \\
\cdot & 21.79 & \cdot & -22.10 \\
-3.51 & \cdot & 5.57 & \cdot \\
\cdot & -22.10 & \cdot & 24.16
\end{array}\right) .
$$

The respective efficiencies for $\theta_{3}, \theta_{(3)}$, and $\theta_{(2)}$ are 66,98 , and 91 percent. The value of the optimality criterion is 0.35553 .

Following the general approach of the Appendix we need to study the polynomial

$$
\begin{aligned}
P(x) & =\frac{1}{6}\left(1, x, x^{2}\right) M_{2}(\xi)^{-1}\left(\begin{array}{c}
1 \\
x \\
x^{2}
\end{array}\right)+\frac{1}{8}\left(1, x, x^{2}, x^{3}\right) M_{3}(\xi)^{-1}\left(\begin{array}{c}
1 \\
x \\
x^{2} \\
x^{3}
\end{array}\right) \\
& =0.94+0.94 x^{2}-3.90 x^{4}+3.02 x^{6} .
\end{aligned}
$$

Since $P$ attains the value one at \pm 1 and at $\pm \sqrt{17 / 117}$, and has local maxima at $\pm \sqrt{17 / 117}$, it is bounded by one on $[-1,1]$. This proves that the design $\xi$ maximizes the geometric mean, on the experimental domain $[-1,1]$.

\subsection{D-Optimal Mixture Designs for $\theta_{(2)}$ and $\theta_{(3)}$, on $\pm 1, \pm 1 / 2,0$}

As an alternative we propose the design that maximizes the same criterion, but restrict the support points of the design to the five Chebyshev points $\pm 1, \pm 1 / 2,0$. The resulting design is

$$
\begin{gathered}
\quad \xi( \pm 1)=.279, \quad \xi( \pm 1 / 2)=.164, \quad \xi(0)=.114, \\
M_{3}(\xi)=\left(\begin{array}{cccc}
1 & \cdot & 0.64 & \cdot \\
\cdot & 0.64 & \cdot & 0.58 \\
0.64 & \cdot & 0.58 & \cdot \\
\cdot & 0.58 & \cdot & 0.56
\end{array}\right), M_{3}(\xi)^{-1}=\left(\begin{array}{cccc}
3.43 & \cdot & -3.80 & \cdot \\
\cdot & 21.88 & \cdot & -22.48 \\
-3.80 & \cdot & 5.92 & \cdot \\
\cdot & -22.48 & \cdot & 24.87
\end{array}\right) .
\end{gathered}
$$


The respective efficiencies for $\theta_{3}, \theta_{(3)}$, and $\theta_{(2)}$ are 64,96 , and 90 percent. The criterion takes the value 0.34974 which is 98 percent of the maximum value of the design in 4.1 . The efficiencies are excellent even though the design is inadmissible (Kiefer 1959).

To compute the design and verify its optimality we procede as indicated in the Appendix. We conjecture the optimal design to be symmetric, $\xi( \pm 1)=w, \xi( \pm 1 / 2)=$ $u, \xi(0)=1-2 w-2 u$, and express the polynomial (4) in terms of $u$ and $w$. If zero belongs to the optimal support then we must have $P(0)=1$, yielding a relation for $u$ in terms of $w$. If further the optimal support point contains \pm 1 then we get $P( \pm 1)=1$, leading to an equation that determines $w$. In summary we obtain

$$
u=\frac{17}{96}-4 w+\sqrt{\left(\frac{17}{96}\right)^{2}+\frac{17}{4} w}, \quad \frac{17}{3 \mu_{2}^{2}}-\frac{10}{\mu_{2}}+\frac{1}{2 w}=0 .
$$

From this, $w$ is computed numerically as .279. The polynomial becomes

$$
P(x)=1+0.78 x^{2}-3.89 x^{4}+3.11 x^{6} .
$$

Now $P( \pm 1)=P( \pm 1 / 2)=P(0)=1$ proves optimality, on the Chebyshev support points $\pm 1, \pm 1 / 2,0$.

\subsection{D-Optimal Mixture Designs for $\theta_{(2)}$ and $\theta_{3}$, on $\pm 1, \pm 1 / 2,0$}

The previous objective function is a mixture of two D-criteria for two different models, the second-degree and the third-degree model. As an alternative approach one may embed the second-degree model in the third-degree model (Atkinson 1972). Hence $\theta_{(2)}$ no longer is the full parameter vector in the model. Rather, it is considered as a subvector of $\theta_{(3)}$.

In the third-degree model, the information matrix for $\theta_{(2)}$ is $A_{11}-A_{12} A_{22}^{-1} A_{21}$, where $A=M_{3}(\xi)$ is partitioned with a top left $3 \times 3$ subblock $A_{11}=M_{2}(\xi)$. That is, the information matrix for $\theta_{(2)}$ now is a difference, the information matrix $A_{11}$ of the seconddegree model minus a 'penalty term' $A_{12} A_{22}^{-1} A_{21}$ which reflects as the loss of information due to fitting the additional parameter $\theta_{3}$.

We now maximize the geometric mean of the D-information for $\theta_{(2)}$ and the information for $\theta_{3}$,

$$
\left\{\left[\operatorname{det}\left(A_{11}-A_{12} A_{22}^{-1} A_{21}\right)\right]^{1 / 3}\left[A_{22}-A_{21} A_{11}^{-1} A_{12}\right]\right\}^{1 / 2}
$$

on the Chebyshev points $\pm 1, \pm 1 / 2,0$. We obtain

$$
\xi( \pm 1)=.168, \quad \xi( \pm 1 / 2)=.332
$$




$$
M_{3}(\xi)=\left(\begin{array}{cccc}
1 & \cdot & 0.50 & \cdot \\
\cdot & 0.50 & \cdot & 0.38 \\
0.50 & \cdot & 0.38 & \cdot \\
\cdot & 0.38 & \cdot & 0.35
\end{array}\right), M_{3}(\xi)^{-1}=\left(\begin{array}{cccc}
3.01 & \cdot & -4.00 & \cdot \\
\cdot & 11.05 & \cdot & -12.04 \\
-4.00 & \cdot & 7.96 & \cdot \\
\cdot & -12.04 & \cdot & 16.00
\end{array}\right) .
$$

The respective efficiencies for $\theta_{3}, \theta_{(3)}$, and $\theta_{(2)}$ are 100,94 , and 75 percent. As the efficiency for $\theta_{3}$ indicates, the present design is practically the same as design 2.1 which is optimal for $\theta_{3}$.

Derivation of the design $\xi$ parallels the steps in 4.2. Again we conjecture the optimal design to be symmetric, $\xi( \pm 1)=w, \xi( \pm 1 / 2)=u, \xi(0)=1-2 w-2 u$. Now the equivalence theorem calls for the investigation of the polynomial

$$
P(x)=\frac{1}{6}\left(1, x, x^{2}, x^{3}\right)\left(\begin{array}{cccc}
\frac{\mu_{4}}{d} & \cdot & -\frac{\mu_{2}}{d} & \cdot \\
\cdot & \frac{4 \mu_{6}}{D}-\frac{3}{\mu_{2}} & \cdot & -\frac{4 \mu_{4}}{D} \\
-\frac{\mu_{2}}{d} & \cdot & \frac{1}{d} & \cdot \\
\cdot & -\frac{4 \mu_{4}}{D} & \cdot & \frac{4 \mu_{2}}{D}-\frac{1}{\mu_{6}}
\end{array}\right)\left(\begin{array}{c}
1 \\
x \\
x^{2} \\
x^{3}
\end{array}\right)
$$

where $d=\mu_{4}-\mu_{2}^{2}$, and $D=\mu_{2} \mu_{6}-\mu_{4}^{2}$.

Suppose $x=0$ is an optimal support point. Then we have $P(0)=1$, entailing a relation to express $u$ in terms of $w$. On the other hand $P(1)=1$ yields an equation that determines $w$. Thus we get

$$
u=\frac{5}{24}-4 w+\sqrt{\left(\frac{5}{24}\right)^{2}+5 w}, \quad \frac{1}{5 \mu_{2}^{2}}-\frac{17}{5 \mu_{2}}-\frac{1}{\mu_{6}}+\frac{2}{w}=0 .
$$

The resulting values $w=.19$ and $u=.44$ are not feasible because the sum $2 w+2 u$ exceeds one. Hence $x=0$ cannot be an optimal support point.

This leaves us with the relation $u=1 / 2-w$. From $P(1)=1$ we calculate $w=.168$, and hence the design $\xi$. Its moments yield the polynomial

$$
P(x)=0.50+5.04 x^{2}-14.73 x^{4}+10.19 x^{6} .
$$

Now $P(0)=0.50<1=P( \pm 1 / 2)=P( \pm 1)$ establishes the optimality of $\xi$, on the five points $\pm 1, \pm 1 / 2,0$.

\section{D-OPTIMAL CONSTRAINT DESIGNS}

The original paper of Stigler (1971) proposed to maximize one criterion while securing some efficiency level for another criterion. The last two designs implement this idea. 


\subsection{D-Optimal Design for $\theta_{(2)}$, Half Efficient for $\theta_{3}$, on $[-1,1]$}

The D-information for $\theta_{(2)}$ in the second-degree model is $\left[\operatorname{det} M_{2}(\xi)\right]^{1 / 3}$. The design maximizing this criterion among those designs on the experimental domain $[-1,1]$ that guarantee 50 percent efficiency for $\theta_{3}$ in the third-degree model, is (Studden 1982)

$$
M_{3}(\xi)=\left(\begin{array}{cccc}
1 & \cdot & 0.64 & \cdot \\
\cdot & 0.64 & \cdot & 0.61 \\
0.64 & \cdot & 0.61 & \cdot \\
\cdot & 0.61 & \cdot & 0.60
\end{array}\right), M_{3}(\xi)^{-1}=\left(\begin{array}{cccc}
3.16 & \cdot & -3.35 & \cdot \\
\cdot & 29.95 & \cdot & -30.14 \\
-3.35 & \cdot & 5.21 & \cdot \\
\cdot & -30.14 & \cdot & 32.00
\end{array}\right) .
$$

The respective efficiencies for $\theta_{3}, \theta_{(3)}$, and $\theta_{(2)}$ are 50, 93, and 94 percent. For all practical purposes the four support points would be considered equispaced in the interval $[-1,1]$.

According to the Appendix the necessary and sufficient condition of the equivalence theorem is in terms of the polynomial $P(x)=\left(1, x, x^{2}, x^{3}\right) N\left(1, x, x^{2}, x^{3}\right)^{\prime}$, where $N$ involves two matrices, one corresponding to the side conditions and the other one to the objective criterion, as well as a Lagrangian multiplier $\alpha$,

$$
N=\alpha\left(\begin{array}{cccc}
\cdot & \cdot & \cdot & \cdot \\
\cdot & 28.40 & \cdot & -30.14 \\
\cdot & \cdot & \cdot & \cdot \\
\cdot & -30.14 & \cdot & 32.00
\end{array}\right)+(1-\alpha)\left(\begin{array}{cccc}
1.05 & \cdot & -1.12 & \cdot \\
\cdot & 0.52 & \cdot & \cdot \\
-1.12 & \cdot & 1.74 & \cdot \\
\cdot & \cdot & \cdot & \cdot
\end{array}\right)
$$

From $P(1)=1$ we obtain $\alpha=.074$, giving

$$
P(x)=0.98+0.52 x^{2}-2.88 x^{4}+2.38 x^{6} .
$$

Now $P(1)=P(0.3236)=1$ and the vanishing of the derivative, $P^{\prime}(0.3236)=0$, imply that on the interval $[-1,1]$ the polynomial $P$ is bounded by one. This proves the desired optimality property of $\xi$.

\subsection{D-Optimal Design for $\theta_{(2)}$, Half Efficient for $\theta_{3}$, on $\pm 1, \pm 1 / 2,0$}

As a final example we take the same criterion as for design 5.1, but again restrict attention to the five Chebyshev points. As a result we obtain the design

$$
\begin{gathered}
\xi( \pm 1)=.292, \quad \xi( \pm 1 / 2)=.123, \quad \xi(0)=.170, \\
M_{3}(\xi)=\left(\begin{array}{cccc}
1 & \cdot & 0.65 & \cdot \\
\cdot & 0.65 & \cdot & 0.60 \\
0.65 & \cdot & 0.60 & \cdot \\
\cdot & 0.60 & \cdot & 0.59
\end{array}\right), M_{3}(\xi)^{-1}=\left(\begin{array}{cccc}
3.28 & \cdot & -3.53 & \cdot \\
\cdot & 29.15 & \cdot & -29.72 \\
-3.53 & \cdot & 5.47 & \cdot \\
\cdot & -29.72 & \cdot & 32.00
\end{array}\right) .
\end{gathered}
$$


The respective efficiencies for $\theta_{3}, \theta_{(3)}$, and $\theta_{(2)}$ are 50, 92, and 93 percent. For all practical purposes these efficiencies are just as good as those of design 5.1. The major difference is that the present design has five support points rather than four.

For the optimality proof we use the notation of Subsection 4.3. The efficiency constraint gives $\mu_{2} / D=32$, whence $u$ is represented in terms of $w$. The polynomial to be studied is $P(t)=\left(1, t, t^{2}, t^{3}\right) N\left(1, t, t^{2}, t^{3}\right)^{\prime}$, with

$$
N=\alpha\left(\begin{array}{cccc}
\cdot & \cdot & \cdot & \cdot \\
\cdot & \frac{\mu_{4}^{2}}{32 D^{2}} & \cdot & -\frac{\mu_{4}}{D} \\
\cdot & \cdot & \cdot & \cdot \\
\cdot & -\frac{\mu_{4}}{D} & \cdot & 32
\end{array}\right)+(1-\alpha)\left(\begin{array}{cccc}
\frac{\mu_{4}}{3 d} & \cdot & -\frac{\mu_{2}}{3 d} & \cdot \\
\cdot & \frac{1}{3 \mu_{2}} & \cdot & \cdot \\
-\frac{\mu_{2}}{3 d} & \cdot & \frac{1}{3 d} & \cdot \\
\cdot & \cdot & \cdot & \cdot
\end{array}\right)
$$

From $P(0)=1$ we obtain a formula for $\alpha$, while $w$ is obtained from $P(1)=1$. In summary we get

$$
u=\frac{4 w}{36 w-1}, \quad \alpha=\frac{6 w^{3}}{(w-1 / 48)(w-1 / 36)}-2 .
$$

With the resulting weights $w=.292$ and $u=.123$ we calculate $\alpha=.086$ and

$$
P(x)=1.00+0.69 x^{2}-3.43 x^{4}+2.75 x^{6} .
$$

Thus $P( \pm 1)=P( \pm 1 / 2)=P(0)=1$ establishes optimality, for the design on the Chebyshev points $\pm 1, \pm 1 / 2,0$.

\section{DISCUSSION}

In the settings discussed in this paper, the design problem relates simultaneously to various models, or various parameter systems, or various optimality criteria. There are many ways to combine information arising from these several sources into a single number. Consequently there are many designs each of which comes with its own good, convincing merits.

The multiplicities to be handled may arise at any one of the following four stages:

- Different models: Depending on the underlying regression functions, a design $\xi$ may give rise to different moment matrices $M_{i}(\xi)$ of different orders $k_{i} \times k_{i}$, for a finite number of models $i=1, \ldots, m$.

- Different parameter systems: In model $i$ the full $k_{i}$-dimensional parameter vector $\theta_{(i)}$ may be of interest, or only an $s_{i}$-dimensional subsystem $K_{i}^{\prime} \theta_{(i)}$, where the $k_{i} \times s_{i}$ coefficient matrix $K_{i}$ is assumed known. 
- Different optimality criteria: Given model $i$ and the parameter system $K_{i}^{\prime} \theta_{(i)}$, different choices are possible for the optimality criterion $\phi_{i}$ to evaluate the information matrix for the parameter system of interest, $C_{K_{i}}\left(M_{i}\right)$. Thus, as a function of the moment matrix $M_{i}$, the objective criterion is a two-fold composition,

$$
\psi_{i}\left(M_{i}\right)=\phi_{i}\left(C_{K_{i}}\left(M_{i}\right)\right) .
$$

- Different averaging criteria: The final step is to average the information quantities $\psi_{1}, \ldots, \psi_{m}$ that originate with the $m$ models, and merge them into a single number $\Phi\left(\psi_{1}, \ldots, \psi_{m}\right)$.

The optimality criteria $\phi$ of greatest interest are the classical D-, A-, and E-criteria. They correspond to maximizing the geometric mean, the harmonic mean, and the minimum of the eigenvalues of the moment matrix. As long as one single model is being investigated, the classical means suffice for all practical purposes.

For the averaging criteria we may similarly select the geometric mean, the harmonic mean, and the minimum of the information quantities $\psi_{i}$. However, when the information from the $m$ models is combined we obtain a grand composition $\phi$,

$$
\phi\left(M_{1} \ldots, M_{m}\right)=\Phi\left(\psi_{1}\left(M_{1}\right), \ldots, \psi_{m}\left(M_{m}\right)\right) .
$$

This terminal composition $\phi$ is not one of the classical means, but does belong to the class of information functions discussed below.

For a unified view of the problem it is therefore imperative to permit a wider class of criteria. The information functions of Pukelsheim (1980) serve this purpose well. By definition, they are required to be

nonnegative,

positively homogeneous,

concave,

nonconstant, and

upper semicontinuous.

For example, the vector means of order $p \in[-\infty, 1]$ are information functions on the vectors $\lambda=\left(\lambda_{1}, \ldots, \lambda_{m}\right)^{\prime}$ in the nonnegative orthant $\mathbb{R}_{+}^{m}$,

$$
\Phi_{p}\left(\lambda_{1}, \ldots, \lambda_{m}\right)= \begin{cases}\left(\frac{1}{m} \sum_{i \leq m} \lambda_{i}^{p}\right)^{1 / p} & \text { for }-\infty<p \leq 1, p \neq 0 \\ \left(\prod_{i \leq m} \lambda_{i}\right)^{1 / m} & \text { for } p=0 \\ \min _{i \leq m}\left\{\lambda_{i}\right\} & \text { for } p=-\infty .\end{cases}
$$


In our examples we have used the geometric mean $\Phi_{0}$ on the quadrant $\mathbb{R}_{+}^{2}$.

Similarly, the matrix means $\phi_{p}$, defined for nonnegative definite $s \times s$ matrices $C$ through

$$
\phi_{p}(C)= \begin{cases}\left(\frac{1}{s} \sum_{i \leq s} C^{p}\right)^{1 / p} & \text { for }-\infty<p \leq 1, p \neq 0 ; \\ (\operatorname{det} C)^{1 / m} & \text { for } p=0 ; \\ \text { smallest eigenvalue of } C & \text { for } p=-\infty ;\end{cases}
$$

are information functions on $\operatorname{NND}(s)$, the cone of nonnegative definite $s \times s$ matrices. Of course, matrix means and vector means are related to each other, in that a matrix mean $\phi_{p}(C)$ on $\operatorname{NND}(s)$ may be reexpressed as a vector mean $\Phi_{p}$ on $\mathbb{R}_{+}^{s}$ applied to the eigenvalues $\left(\lambda_{1}(C), \ldots, \lambda_{s}(C)\right)^{\prime}$ of $C$.

In general, a composition of such means $\Phi\left(\lambda_{1}, \ldots, \lambda_{m}\right)$ and $\phi_{1}\left(C_{1}\right), \ldots, \phi_{m}\left(C_{m}\right)$ fails to produce a classical mean. However, any composition of the form

$$
\phi=\Phi\left(\psi_{1}, \ldots, \psi_{m}\right)
$$

does enjoy all the properties that constitute an information function, provided only that $\Phi$ is an information function on $\mathbb{R}_{+}^{m}$, and $\psi_{i}$ is an information function on $\operatorname{NND}\left(k_{i}\right)$, for all models $i=1, \ldots, m$. This shows that the concept of information functions is wide enough to embrace the classical criteria, and also to permit functional operations such as forming compositions of information functions.

Hence for the type of problems discussed in the present paper a unified view emerges when we employ an Equivalence Theorem of sufficient generality to apply to arbitrary information functions. An appropriate result has been given by Pukelsheim (1980), and is briefly reviewed in the Appendix. Just as in the original Equivalence Theorem of Kiefer and Wolfowitz (1960), the theorem typically leads to one set of equations which implicitly determine the optimal weights - as in 3.2, 4.2, 4.3, 5.2-, while another set determines the optimal support points—see Studden (1982), or Dette (1990).

Three points deserve a final comment. The designs in $4.2,4.3$, and 5.2 , on the five equispaced support points $\pm 1, \pm 1 / 2,0$, are inadmissible (Kiefer 1959). Nevertheless they enjoy excellent efficiencies. This illustrates one virtue of a general appraoch, to calculate the efficiencies explicitly and thus provide numerical, undisputable evidence that the designs not just look good, but do indeed perform well.

Second, all of our examples end up with a design that is symmetric around zero. This demonstrates that symmetry considerations and a reduction by invariance applies to general information functions in the same powerful way it helps with the classical criteria. 
Third, when it comes to combining information, as was done in Sectin 4 for mixture designs, the most sensitive issue is that of scaling. The information quantities to be averaged must somehow be scaled to be represented in comparable units. The sole exception is the geometric mean of the determinant criteria, which is why we have chosen them for the examples in the present paper.

Namely, the design ordering that originates with the determinant criterion $\phi_{0}$ is invariant under nonsingular affine transformations (Gaffke 1981). A similar order invariance pertains to the geometric mean $\Phi_{0}$. The reason is that $\Phi_{0}$ is homogeneous separately in each variable $\lambda_{i}$. This is not true for other information functions on $\mathbb{R}_{+}^{m}$. Therefore the determinant criteria, and geometric means thereof, lead to the same optimal design irrespective of how the regression functions, and hence the moment matrices, are scaled. This provides a strong argument in their favor.

\section{APPENDIX. EQUIVALENCE THEOREM}

The Equivalence Theorem concentrates on moment matrices rather than on the set $\Xi$ of all designs $\xi$. We have encountered the following sets $\mathcal{M}$ of moment matrices:

$$
\begin{aligned}
& \mathcal{M}=\left\{\frac{1}{2} M_{2}\left(\xi_{0}\right)+\frac{1}{2} M_{2}(\xi): \xi \in \Xi\right\} \quad \subseteq \mathrm{NND}(3), \\
& 4.3 \mathcal{M}=\left\{M_{3}(\xi): \xi \in \Xi\right\} \quad \subseteq \operatorname{NND}(4) \text {, }
\end{aligned}
$$$$
\text { 5.1, 5.2 } \mathcal{M}=\left\{M_{2}(\xi): \xi \in \Xi,\left(M_{3}(\xi)^{-1}\right)_{44} \leq 32\right\} \subseteq \mathrm{NND}(3) .
$$

Each of these sets is convex and compact, which are the only two properties called for by the Equivalence Theorem.

As pointed out in the Discussion, all the optimality criteria considered are information functions $\phi$. As a substitute for the notion of a gradient, or a subgradient, it proves to be advantageous to introduce the polar information function $\phi^{\infty}$ by defining, for any nonnegative matrix $D$,

$$
\phi^{\infty}(D)=\inf _{C>0} \frac{\operatorname{trace} C D}{\phi(C)}
$$

where the notation $C>0$ designates positive definiteness of $C$. For example, the polar functions of the matrix means $\phi_{p}$ on $\operatorname{NND}(s)$ are known to be $\phi_{p}^{\infty}=s \phi_{q}$, where the numbers $p$ and $q$ are conjugate, $p+q=p q$.

For the sake of simplicity we assume the moment matrix $M=M(\xi)$ that is checked for optimality to be positive definite. 
Theorem. Let the set $\mathcal{M}$ of moment matrices be convex and compact, and let the optimality criterion $\phi$ be an information function.

Then a design $\xi$ with positive definite moment matrix $M=M(\xi)$ in $\mathcal{M}$ maximizes the criterion $\phi$ over $\mathcal{M}$ if and only if there exists a nonnegative solution $N$ of the equation

$$
\phi(M) \phi^{\infty}(N)=\operatorname{trace} M N=1
$$

which satisfies

$$
\text { trace } A N \leq 1 \text { for all } A \in \mathcal{M}
$$

For a proof see Pukelsheim (1980). Equation (P) relates to the polar function $\phi^{\infty}$, and is called the polarity equation. Inequality $(\mathrm{N})$ requires the matrix $N$ to be normal to the set $\mathcal{M}$ at $M$, and is called the normality inequality.

We have already mentioned that the matrix means $\phi_{p}$ have polars proportional to $\phi_{q}$. This and related results make it usually easy to solve the polarity equation $(\mathrm{P})$, and display the solution(s) $N$ in terms of $M$.

The point is to verify the normality inequality $(\mathrm{N})$. For a third-degree model the set $\mathcal{M}$ is generated by the rank one matrices $f(x) f(x)^{\prime}$ with $x \in[-1,1]$, where $f(x)$ is the power vector $\left(1, x, x^{2}, x^{3}\right)^{\prime}$. Hence the left hand side of the normality inequality $(\mathrm{N})$ turns into a polynomial $P(x)$,

$$
\operatorname{trace} f(x) f(x)^{\prime} N=f(x)^{\prime} N f(x)=\left(1, x, x^{2}, x^{3}\right)^{\prime} N\left(\begin{array}{c}
1 \\
x \\
x^{2} \\
x^{3}
\end{array}\right)=P(x) \text {. }
$$

Thus (N) boilds down to calculate the polynomial $P(x)$ that comes with the optimality candidate $M$, and check whether on $[-1,1]$ it is bounded by one. The proofs of our examples all follow this pattern.

[First Version; 5 July 1991.] 


\section{REFERENCES}

Atkinson, A.C. (1972), "Planning experiments to detect inadequate regression models," Biometrika, 59, 275-293.

Atkinson, A.C., and Cox, D.R. (1974), "Planning experiments for discriminating between models," Journal of the Royal Statistical Society, Series B 36, 321-334. "Discussion on the paper by Dr Atkinson and Professor Cox," ibidem, 335-348.

Atkinson, A.C., and Fedorov, V.V. (1975a), "The design of experiments for discriminating between two rival models," Biometrika, 62, 57-70.

(1975b), "Optimal design: Experiments for discriminating between several models," Biometrika, 62, 289-303.

Box, G.E.P., and N.R. Draper (1959). "A basis for the selection of a response surface design," Journal of the American Statistical Association, 54, 622-654.

Bunke, H., and Bunke, O. (1986), Statistical Inference in Linear Models. Statistical Methods of Model Building, Volume I, Chichester: Wiley.

Chaloner, K. (1984), "Optimal Bayesian experimental design for linear models," The Annals of Statistics, 12, 283-300, "Correction," ibidem, 13, 836.

Cook, R.D., and Nachtsheim, C.J. (1982), "Model robust, linear-optimal designs," Technometrics, 24, 49-54.

Covey-Crump, P.A.K., and Silvey, S.D. (1970), "Optimal regression designs with previous observations," Biometrika, 57, 551-566.

Dette, H. (1990), "A generalization of $D$ - and $D_{1}$-optimal designs in polynomial regression," The Annals of Statistics, 18, 1784-1804.

(1991), "A mixture of the $D$ - and $D_{1}$-optimality criterion in polynomial regression," Technical Report \#91-23C, 21 pages, Department of Statistics, Purdue University.

Gaffke, N. (1981). "Some classes of optimality criteria and optimal designs for complete two-way layouts," The Annals of Statistics, 9, 893-898.

Hunter, W.G., and Reiner, A.M. (1965), "Designs for discriminating between two rival models," Technometrics, 7, 307-323. 
Kiefer, J.C. (1959), "Optimum experimental designs," Journal of the Royal Statistical Society, Series B 21, 272-304, "Discussion on Dr Kiefer's paper," ibidem, 304-319. (1974), "General equivalence theory for optimum designs (approximate theory)," The Annals of Statistics, 2, 849-879.

Kiefer, J.C., and Wolfowitz, J. (1959), "Optimum designs in regression problems," The Annals of Mathematical Statistics, 30, 271-294.

(1960), "The equivalence of two extremum problems," Canadian Journal of Mathematics, 12, 363-366.

Lau, T.-S. (1988), "D-optimal designs on the unit q-ball," Journal of Statistical Planning and Inference, 19, 299-315.

Lau, T.-S., and Studden, W.J. (1985), "Optimal designs for trigonometric and polynomial regression using canonical moments," The Annals of Statistics, 13, 383-394.

Läuter, E. (1974), "Experimental design in a class of models," Mathematische Operationsforschung und Statistik, 5, 379-398.

(1976), "Optimal multipurpose designs for regression models," Mathematische Operationsforschung und Statistik, 7, 51-68.

Lee, C.M.-S. (1987), "Constrained optimal designs for regression models," Communications in Statistics: Theory and Methods, 16, 765-783.

(1988), "Constrained optimal designs," Journal of Statistical Planning and Inference, 18, 377-389.

Lim, Y.B., and Studden, W.J. (1988), "Efficient $D_{s}$-optimal design for multivariate polynomial regression on the q-cube," The Annals of Statistics, 16, 1225-1240.

Pukelsheim, F. (1980), "On linear regression designs which maximize information," Journal of Statistical Planning and Inference, 4, 339-364.

(1992), Optimality Theorey of Experimental Designs in Linear Models, New York: Wiley.

Stigler, S.M. (1971), "Optimal experimental design for polynomial regression," Journal of the American Statistical Association, 66, 311-318.

Studden, W.J. (1968), "Optimal designs on Tchebycheff points," The Annals of Mathematical Statistics, 39, 1435-1447. 
(1982), "Some robust-type D-optimal designs in polynomial regression," Journal of the American Statistical Association, 77, 916-921.

Welch, W.J. (1982), "Branch-and-bound search for experimental designs based on $D$ optimality and other criteria,"

Technometrics, 24, 41-48.

Wynn, H.P. (1977), "Optimum designs for finite populations sampling," in Statistical Decision Theory and Related Topics II, eds. S.S. Gupta and D.S. Moore, New York: Academic Press, pp. 471-478.

(1982), "Optimum submeasures with application to finite population sampling," in Statistical Decision Theory and Related Topics III (vol. 2), eds. S.S. Gupta and J.O. Berger, New York: Academic Press, pp. 485-495. 